\title{
An induction heating device using planar coil with high amplitude alternating magnetic fields for magnetic hyperthermia
}

\author{
Zuhe Wu, Zihang Zhuo, Dongyang Cai, Jian'an Wu, Jie Wang and Jintian Tang* \\ Key Laboratory of Particle Technology and Radiation Imaging, Ministry of Education, Department of \\ Engineering Physics, Tsinghua University, Beijing, China
}

\begin{abstract}
.
BACKGROUND: Induction heating devices using the induction coil and magnetic nanoparticles (MNPs) are the way that the magnetic hyperthermia is heading.

OBJECTIVE: To facilitate the induction heating of in vivo magnetic nanoparticles in hyperthermia experiments on large animals.

METHODS: An induction heating device using a planar coil was designed with a magnetic field frequency of $328 \mathrm{kHz}$. The coil's magnetic field distribution and the device's induction heating performance on different concentrations of magnetic nanoparticles were measured.

RESULTS: The alternating magnetic field produced in the axis position $165 \mathrm{~mm}$ away from the coil center is $40 \mathrm{Gs}$ in amplitude; magnetic nanoparticles with a concentration higher than $80 \mathrm{mg} . \mathrm{mL}^{-1}$ can be heated up rapidly.

CONCLUSION: Our results demonstrate that the device can be applied not only to in vitro and in small animal experiments of magnetic hyperthermia using MNPs, but also in large animal experiments.
\end{abstract}

Keywords: Magnetic hyperthermia, magnetic nanoparticles, planar coil, power abortion, induction heating

\section{Introduction}

Magnetic hyperthermia is a novel method of cancer treatment using magnetic nanoparticles (MNPs) implanted in tumor tissues to kill cancerous cells by producing heat in an alternating magnetic field (AMF) by the magnetization losses from hysteresis, eddy current, and/or magnetic relaxation effects [13]. Induction heating devices for generating an AMF generally use a ferromagnetic core structure or induction coil structure [4-7]. The devices employing ferromagnetic core have been used in clinical experiments [6-8] but it is difficult for them to further improve the frequency and intensity of the AMF due to the large volume of the core and significant self-heating inside it. With smaller volume and less self-heating, the induction coil, which generally based on resonant inverters, was used and researched more and more frequently [5|9-11].

\footnotetext{
${ }^{*}$ Corresponding author: Jintian Tang, Key Laboratory of Particle Technology and Radiation Imaging, Ministry of Education, Department of Engineering Physics, Tsinghua University, Beijing, China. Tel.: +86 010 62796769; Fax: +86 010 62789237; E-mail: tangjt@mail.tsinghua.edu.cn.
} 
Solenoid coil and planar coil have both been reported in hyperthermia induction heating devices. Lacroix et al. [12], Ivkov et al. [5] and Garaio et al. [13] employed solenoid coil in their hyperthermia applicator with a diameter of $11 \mathrm{~mm}, 36 \mathrm{~mm}$ and $15 \mathrm{~mm}$, respectively. Similarly, a solenoid coil of $60 \mathrm{~mm}$ height, $50 \mathrm{~mm}$ diameter and 25 turns was described by Cano et al. [14] and the maximum magnetic flux intensity reached 150 Gs. Piao et al. [15] reported an induction heater that could heat samples both in vertical and in horizontal orientations and used a solenoid coil of $60 \mathrm{~mm}$ height and $40 \mathrm{~mm}$ inner diameter to generate a magnetic field of $220 \mathrm{Gs}$ and $156 \mathrm{kHz}$. These devices were mainly designed for vitro and small animal experiments, but were inappropriate in the cases of large animal or clinical applications. For example, in the liver tumor magnetic hyperthermia experiment in rats, it was difficult to locate rats with a diameter larger than $50 \mathrm{~mm}$ into the solenoid coil. Moreover, the magnetic field decreases so rapidly as the distance from the coil surface increases that it is hard to heat deep located MNPs in vivo. Akiyama and Kawasaki et al. [16] successfully applied a system with coaxial planar coils to heat a metal stent in the treatment of esophageal cancer patients, but its magnetic field was too weak to heat MNPs. Durr et al. [17] designed an induction heating system with two parallel planar coils inside which MNPs were placed. The pitch of the two coils was $40 \mathrm{~mm}-100 \mathrm{~mm}$ and when the pitch was $100 \mathrm{~mm}$, the maximum magnetic field intensity in the center of the coil could reach 85 Gs. However, owing to the largest pitch of $100 \mathrm{~mm}$, large animals such as pigs could not be placed appropriately. Nagae et al. [18] used a single planar coil induction heating device to conduct magnetic particle experiments in pigs. A single flat pancake-like coil composed of 6 turns was used, and the maximum magnetic flux intensity about $10 \mathrm{~mm}$ from the coil axis was $180 \mathrm{Gs}$. Unfortunately, no more technical and building details were provided in this reference. However, it provided a perspective for the coil design of an induction heater device for magnetic hyperthermia. Compared with the biplane coil designed by Durr et al. [17], the structure and circuit of single planar was simpler. Besides, with the advantage of volume and self-structure, the heating of MNPs in large animal experiments could be realized more easily in planar coil than solenoid coil.

To facilitate the heating of MNPs in deep tumors of large animals, based on single planar coil, we designed an induction heating device of $300 \mathrm{kHz}$, and this study described the design progress in detail. In addition, the electrical parameters and distribution of magnetic field of the device was measured and the heating performance of MNPs was tested.

\section{Materials and methods}

\subsection{Design of induction heater device}

As Fig. 1(a) presented, the device mainly included three parts: the three-phase input rectifier; the BUCK converter; and the high frequency inverter circuit and resonant circuit. The current entered the BUCK converter from the three-phase power supply after rectification and filtering. The BUCK converter used the DC Buck chopper circuit operating in continuous conduction mode [19-21]. The heating power was adjusted by changing the duty cycle of the IGBT control signals driving the BUCK circuit. Switching transistors of full-bridge inverter circuit were in parallel with reversed SiC Schottky diode unit to provide follow current when the inverter voltage was reversed. The snubber circuit with series connection of $\mathrm{R}$ and $\mathrm{C}$ was used for each branch. The output of the inverter was coupled to the $\mathrm{LC}$ resonant circuit through a high frequency transformer with a turn ratio of 15:1 (Fig. 1(b)).

The planar coil, wound by six turns around a hollow copper pipe, had an inductance (Lr) of about $2.7 \mathrm{uH}$ (Fig. 1(c)). The capacity $\mathrm{Cr}$ of the capacitor bank was set as $0.10 \mathrm{uF}$ to get a resonant frequency 


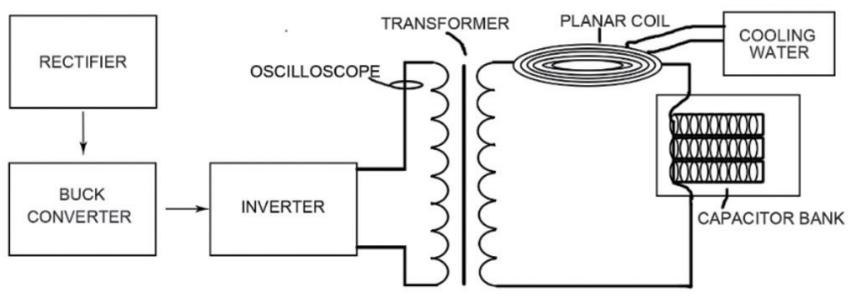

(a)

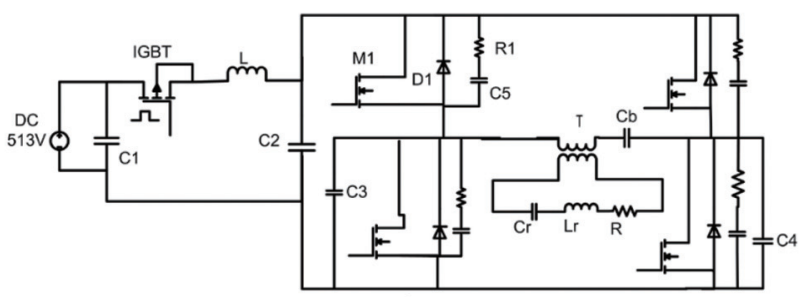

(b)

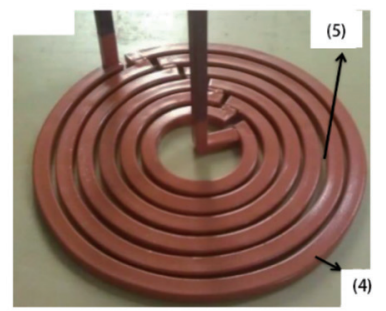

(c)

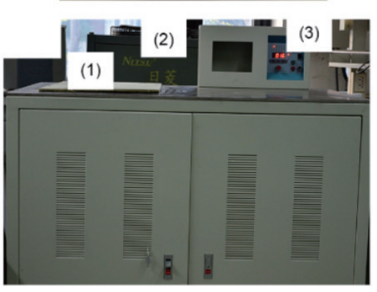

(d)

Fig. 1. Design details of device. (a) Block diagram of device (b) BUCK converter, inverter and resonance circuit (c) The single planar coil: The outer diameter of was $32 \mathrm{~cm}$, the cross-sectional area of copper pipe was $30 * 25 \mathrm{~mm}$, the thickness of the copper pipe was $2 \mathrm{~mm}$, and the gap between each turn was $8 \mathrm{~mm}-12 \mathrm{~mm}$ (d) Induction heater device assembled. The planar coil was below the glass partition (1). The control and display panel (3) was used to regulate device power and show the current value on the three-phase line. The chiller (2) was outside the equipment.

of $300 \mathrm{kHz}$. We used 27 capacitor banks with capacitance value of $0.33 \mathrm{uF}$ and voltage withstand value of $700 \mathrm{~V}$, which were connected in accordance with the structure of the 9-in-series and 3-in-parallel. In addition, an external cooling system with maximum water flow of $102 \mathrm{~cm}^{2} / \mathrm{s}$ was adopted to cool the planar coil.

\subsection{Simulation and measurement of the distribution of magnetic field}

A model of the single planar coil was established in Computer Simulation Technology (CST) according to the planar coil described in Fig. 1(c). The material of the model was copper and the environment space was air.

After the coil winding, the coil's DC resistance R was $59 \mathrm{~m} \Omega$. When the output voltage of BUCK circuit was $510 \mathrm{~V}$, the theoretical maximum current of the secondary coil was $576 \mathrm{~A}$. In view of stability and safety, it is generally downgraded in practical application. Thus, the model coil carrier was assigned $500 \mathrm{~A}$ when simulating the magnetic field. In order to verify the distribution of the magnetic field, the current of the coil was adjusted to $500 \mathrm{~A}$. The magnetic field above the planar coil was measured with three-dimensional and one-dimensional magnetic field measuring probes. The measuring point was located on the coil axis.

\subsection{Measurement of electrical parameters}

The current transformer OPCT16AL (input-output ratio 3000:1) was used to measure the coil current. Since the maximum allowance current of the transformer was $200 \mathrm{~A}$ and was less than the maximum current flowing in the coil, the transformer was placed over the high-frequency transformer primary current during measurement (as shown in Fig. 1(a)) and the coil current was calculated according to the 


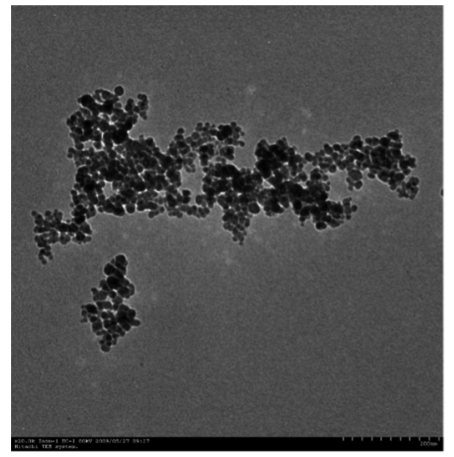

(a)

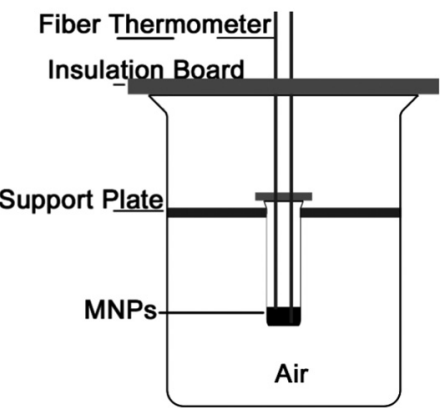

(b)

Fig. 2. (a) Electron micrograph of magnetic particles (b) Schematic diagram of induction heating: $2 \mathrm{~mL}$ of magnetic particle solution were put into each test tube, the tubes were placed in a sealed beaker with insulation panels in order to reduce heat loss.

turn ratio. The transformer was side-jointed with a $5 \Omega$ load for the second time, and MSO-X 2024A (Agilent Technologies) measured the load voltage.

Adjusting the panel reading to change the input power tested the power regulation linearity of the system; the coil current was measured synchronously.

\subsection{Heating experiment of MNPs in vitro}

The MNPs with the size of about $10 \mathrm{~nm}$ presented in Fig. 2(a) were synthesized by means of chemical co-precipitation [22]. The MNPs concentrations were set at 100, 80, 60 and $40 \mathrm{mg} \cdot \mathrm{mL}^{-1}$. During the experiment, a breaker was placed in the central plane of planar coil as displayed in Fig. 1(d), and the heating power was adjusted until the magnetic flux intensity of the upper surface of MNPs reached about 40 Gs. Dual-channel fiber thermometers were used to measure the temperature during the experiment. One was placed in the upper end of the MNPs and the other was placed in the lower end, see Fig. 2(b). The temperature of the MNPs was the average temperature value of the two channels.

\section{Results and discussion}

\subsection{Measurement of the coil frequency and current}

Figure 3(a) showed the resonant frequency of the resonant circuit was about $328 \mathrm{kHz}$ which was slightly larger than $300 \mathrm{kHz}$. It was difficult to make the total capacitance value after the series or parallel combinations match the same with the theoretical value of $0.10 \mathrm{uF}$ because the capacitance of the single capacitor in the oscillation circuit was $0.33 \mathrm{uF}$ and the limit of voltage withstand and current must be considered when designing the capacitor bank connection. In addition, due to limitations in the winding process, there was an error between the actual and theoretical values of the inductance of the coil. As a result, the resonant frequency was not equal to $300 \mathrm{kHz}$. However, we still consider this error of frequency acceptable for the actual frequency not the designed frequency would be suggested to the user of this device in simulation and calculation of the power absorption of MNPs which was related to the frequency of the magnetic field [7]23-25].

The maximum current of the coil reached about $528 \mathrm{~A}$, resulting in a maximum device power of $17.6 \mathrm{~kW}$. As mentioned earlier, we adjusted the power by changing the output voltage of the BUCK 


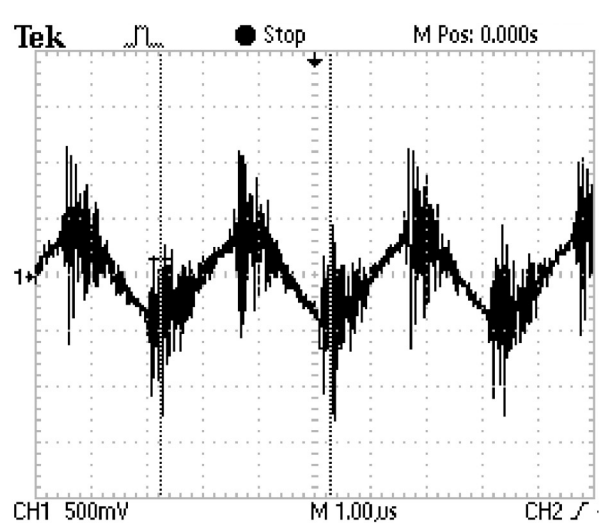

(a)

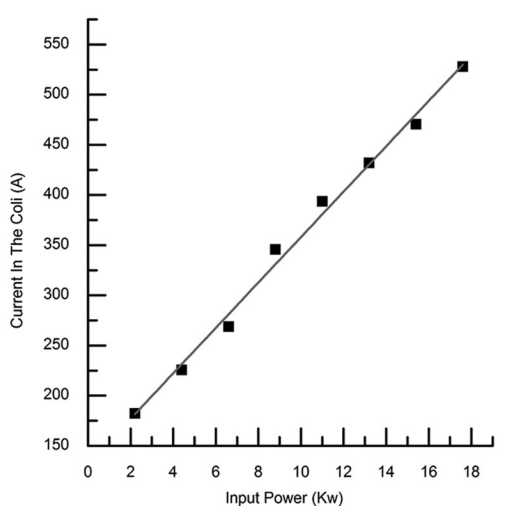

(b)

Fig. 3. (a) Image of voltage signal converted on the load from the output current signal of the transformer in the oscilloscope (b) Current values of the coil under different device power.

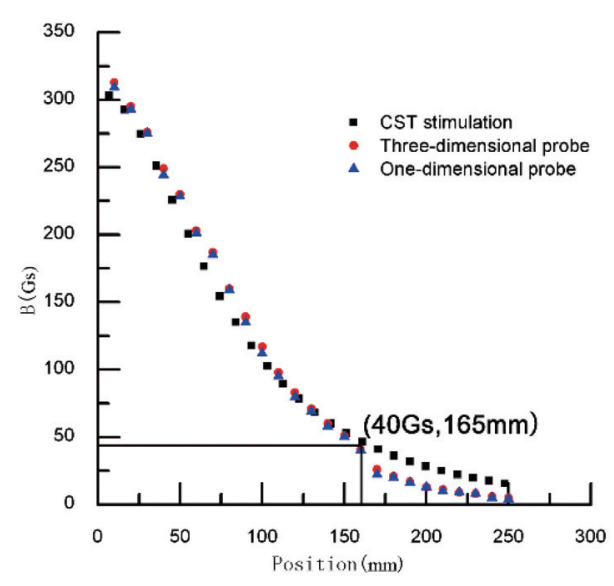

(a)

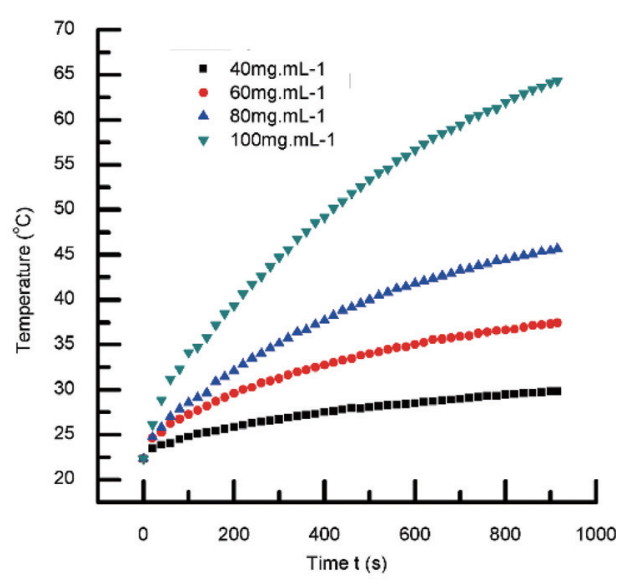

(b)

Fig. 4. (a) Measurement of the magnetic field intensity (b) Temperature curves of the heated samples of different concentrations.

circuit. In Fig. 3(b) the linear fitting result of $R^{2}=0.992$ indicated a good linear correlation with the coil current and the input power and the coil current could be adjusted approximately linearly by adjusting the reading of the panel.

\subsection{The distribution of magnetic field}

Figure 4(a) showed that the magnetic field direction of the coil axis was approximately perpendicular to the coil plane, so the measured values of the two probes were approximately the same and were in good agreement with the simulation results.

Compared with the device used by Nagae et al. [18], we significantly increased the maximum magnetic field $10 \mathrm{~mm}$ away from the coil plane on the axis. Thus, our device may improve the heating depth for MNPs in the tumor in vivo. To enable rapid heating of the magnetic particles, the minimum intensity of the external magnetic field was recommended to be set greater than $40 \mathrm{Gs}$ [3|26|27], so the maximum heating depth of MNPs in the tumor on the axis of the device could reach about $165 \mathrm{~mm}$. The more 
uniform the magnetic field was, the better the temperature stability of MNPs was [28]. The measurement and simulation results indicated that the magnetic field intensity decreased rapidly with the increasing distance from coil surface, producing adverse effects on the temperature stability of MNPs. The problem of the magnetic field's non-uniformity could be improved by using a biplane coil as Durr et al. [17] did in the cost of increasing complexity of system. Therefore, it is still necessary to study the biplane coil structure in the case of minimizing the design complexity.

\subsection{Heating performance of MNPs}

The experiment results of particles of different concentrations are presented in Fig. 4(b). In the magnetic field of about $40 \mathrm{Gs}$ and $328 \mathrm{kHz}$, the greater the particle concentration, the more rapidly the temperature rose. The vitro experiment demonstrated that the MNPs with low concentrations heated slowly. In order to quickly reach the temperature $46^{\circ} \mathrm{C}[7|28| 29]$, we recommend that the concentration of the particles greater than $80 \mathrm{mg} \cdot \mathrm{mL}^{-1}$. As the tumor tissue has better thermal conductivity than air, and the blood circulation removes the heat generated by the media, the rise of temperature of MNPs in tumor tissue will be much slower than the results in in vitro experiment. Therefore, the MNPs concentration and magnetic field intensity in animal experiments must be chosen carefully.

In MNPs experiments, large animals are usually placed on the surface of coil. According to 3.2, we achieved a magnetic field of $40 \mathrm{Gs}$ in the position about $165 \mathrm{~mm}$ above the planar coil plane; thus, as demonstrated by Fig. 4(b), MNPs with suitable concentrations injected into nearly any part of large animals can be heated effectively. Moreover, samples could be placed more easily compared with induction heater device using a solenoid coil.

\section{Conclusions}

The design and characterization of an induction heater device for magnetic hyperthermia in large animals has been described in detail. One feature of our device is the single planar coil, which makes it more convenient and easy to place large animals than the solenoid coil. Another feature of our device is high amplitude alternating magnetic fields on the surface of the coil. The magnetic field distribution and MNPs experiments in vitro show that the device can heat MNPs at a depth of about $165 \mathrm{~mm}$ and is suitable for MNPs experiments in almost any occasion, such as tube experiments and small animal and large animal experiments. In future research, we will gradually improve the efficiency of our equipment and study new coil topology.

\section{Acknowledgments}

This work was supported by the Beijing Muni-cipal Science and Technology Commission (Z1111000 67311053), and the Technology Support Program of China (2012BAI15B04). We also acknowledge CST China Ltd for the trial version of CST studio suite.

\section{References}

[1] Cherukuri P, Glazer E S, Curley S A. Targeted hyperthermia using metal nanoparticles. Advanced Drug Delivery Reviews. 2010; 62(3): 339-345. 
[2] Asin L, Ibarra M R, Tres A, et al. Controlled cell death by magnetic hyperthermia: effects of exposure time, field amplitude, and nanoparticle concentration. Pharmaceutical Research. 2012; 29(5): 1319-1327.

[3] Pankhurst Q A, Thanh N T K, Jones S K, et al. Progress in applications of magnetic nanoparticles in biomedicine. Journal of Physics D: Applied Physics. 2009; 42(22): 224001.

[4] Salunkhe A B, Khot V M, Pawar S H. Magnetic hyperthermia with magnetic nanoparticles: A status review. Current Topics in Medicinal Chemistry. 2014; 14(5): 572-594.

[5] Ivkov R, DeNardo S J, Daum W, et al. Application of high amplitude alternating magnetic fields for heat induction of nanoparticles localized in cancer. Clinical Cancer Research. 2005; 11(19): 7093s-7103s.

[6] Jordan A, Scholz R, Wust P, et al. Magnetic fluid hyperthermia (MFH): Cancer treatment with AC magnetic field induced excitation of biocompatible superparamagnetic nanoparticles. Journal of Magnetism and Magnetic Materials. 1999; 201(1): 413-419.

[7] Jordan A, Scholz R, Maier-Hauff K, et al. Presentation of a new magnetic field therapy system for the treatment of human solid tumors with magnetic fluid hyperthermia. Journal of Magnetism and Magnetic Materials. 2001; 225(1): 118-126.

[8] Johannsen M, Thiesen B, Wust P, et al. Magnetic nanoparticle hyperthermia for prostate cancer. International Journal of Hyperthermia. 2010; 26(8): 790-795.

[9] Oleson J R. A review of magnetic induction methods for hyperthermia treatment of cancer. Biomedical Engineering, IEEE Transactions on. 1984; (1): 91-97.

[10] Jian L, Shi Y, Liang J, et al. A novel targeted magnetic fluid hyperthermia system using HTS coil array for tumor treatment. Applied Superconductivity, IEEE Transactions on. 2013; 23(3): 4400104-4400104.

[11] Boadi A, Tsuchida Y, Todaka T, et al. Designing of suitable construction of high-frequency induction heating coil by using finite-element method. Magnetics, IEEE Transactions on. 2005; 41(10): 4048-4050.

[12] Lacroix L M, Carrey J, Respaud M. A frequency-adjustable electromagnet for hyperthermia measurements on magnetic nanoparticles. Review of Scientific Instruments. 2008; 79(9): 093909-093909-5.

[13] Garaio E, Collantes J M, Plazaola F, et al. A multifrequency eletromagnetic applicator with an integrated AC magnetometer for magnetic hyperthermia experiments. Measurement Science and Technology. 2014; 25(11): 115702.

[14] Cano M E, Barrera A, Estrada J C, et al. An induction heater device for studies of magnetic hyperthermia and specific absorption ratio measurements. Review of Scientific Instruments. 2011; 82(11): 114904.

[15] Piao D, Le K, Saunders D, et al. Development of a vertically and horizontally applicable multi-frequency alternatingmagnetic-field device for hyperthermia of glioma in rodent model using iron oxide based nanoparticles[C]//biomedical optics. Optical Society of America. 2012; BSu3A. 2.

[16] Akiyama S, Kawasaki S, Kodera Y, et al. A new method of thermo-chemotherapy using a stent for patients with esophageal cancer. Surgery Today. 2006; 36(1): 19-24.

[17] Dürr S, Schmidt W, Janko C, et al. A novel magnetic field device for inducing hyperthermia using magnetic nanoparticles. Biomedical Engineering/Biomedizinische Technik. 2013.

[18] Nagae H, Nagano I, Yagitani S, et al. Development of new cancer treatment using approved MRI contrast agent and induction heating device[C]//general assembly and scientific symposium, 2011 XXXth URSI. IEEE. 2011: 1-4.

[19] Liu S L, Liu J, Li Y, and Mao H. Equivalent-resistance based on the analysis of inductor-disconnected discharge behavior for intrinsic safety Buck converters. Journal of Coal Science and Engineering (China). 2008; 14, 485-488.

[20] Li Y, Zhang J. LC filter using high-power switching converter buck. Modern Electronic Technology (China). 2012; 35(12): 137-142.

[21] Liu J. Single-inductor dual-output switching converter under continuous conduction mode. Telecom Power Technologies (China). 2010; 27(1): 19-22.

[22] Bai L Z, Zhao D L, Xu Y, et al. Inductive heating property of graphene oxide-Fe $3 \mathrm{O} 4$ nanoparticles hybrid in an AC magnetic field for localized hyperthermia. Materials Letters. 2012; 68: 399-401.

[23] Haider S A, Cetas T C, Wait J R, et al. Power absorption in ferromagnetic implants from radiofrequency magnetic fields and the problem of optimization. Microwave Theory and Techniques, IEEE Transactions on. 1991; 39(11): 1817-1827.

[24] Zhuo Z H, Wang J, Zhai W M, et al. Numerical modeling and simulation of temperature distribution uncertainty subject to ferromagnetic thermoseeds hyperthermia. Chinese Science Bulletin. 2014; 59(12): 1317-1325.

[25] Li X, Gao X, Tang J. Heating effects of heat seed in muscle of magnetic induction. Technology Review (China). 2010; 28(19): 77-80.

[26] Hu R, Liu X, Tang J. Thermal effects of magnetic fluid for hyperthermia cancer in vitro. Chinese Journal of Minimally Invasive Surgery (China). 2007; 7(11): 1049-1050.

[27] Wu Y. Tumor-induced hyperthermia alternating magnetic field generating prototype device developed. Dissertation, Nanjing: Southeast University of China; 2004.

[28] Hergt R, Dutz S, Müller R, et al. Magnetic particle hyperthermia: Nanoparticle magnetism and materials development for cancer therapy. Journal of Physics: Condensed Matter. 2006; 18(38): S2919.

[29] Berry C C. Progress in functionalization of magnetic nanoparticles for applications in biomedicine. Journal of Physics D: Applied Physics. 2009; 42(22): 224003. 